\title{
Average Channel Capacity of Amplify-and-forward MIMO/FSO Systems Over Atmospheric Turbulence Channels
}

\author{
Duong Huu Ai \\ Faculty of Electronics and Telecommunications, Korea Vietnam Friendship Information Technology College, Vietnam \\ Department of Aerospace Electronics, School of Electronics and Telecommunications, Hanoi University of Science and \\ Technology, Vietnam
}

\begin{tabular}{l} 
Article Info \\
\hline Article history: \\
Received Oct 6, 2017 \\
Revised Jul 18, 2018 \\
Accepted Aug 1, 2018 \\
\hline
\end{tabular}

\section{Keyword:}

Amplify-and-forward Atmospheric turbulence Average channel capacity Average spectral efficiency Free-space optical

\begin{abstract}
In amplify-and-forward (AF) relay channel, when the direct link between source and destination terminals is deeply faded, the signal from the source terminal to the destination terminal propagates through the relay terminals, each of which relays a signal received from the previous terminal to the next terminal in series. This paper, we theoretically analyze the performance of multiple-input multiple-output (MIMO) AF free-space optical (FSO) systems. The AF-MIMO/FSO average channel capacity (ACC), which is expressed in terms of average spectral efficiency (ASE) is derived taking into account the atmospheric turbulence effects on the MIMO/FSO channel. They are modeled by log-normal and the gamma-gamma distributions for the cases of weak-to-strong turbulence conditions. We extract closed form mathematical expression for the evaluation of the ACC and we quantitatively discuss the influence of turbulence strength, link distance, different number of relay stations and different MIMO configurations on it.
\end{abstract}

Copyright (c) 2018 Institute of Advanced Engineering and Science. All rights reserved.

\section{Corresponding Author:}

Duong Huu Ai,

Faculty of Electronics and Telecommunications,

Korea Vietnam Friendship Information Technology College,

136 Tran Dai Nghia St., Danang, Vietnam.

Email: aidh@viethanit.edu.vn

\section{INTRODUCTION}

The necessity of a cost-effective, license-free, high security and high bandwidth access technique has lead to a continuous research and commercial interest in optical wireless communication systems [1]-[3]. One of major degradations to the performance of FSO communications is the influence of atmospheric turbulence caused by variations in the refractive index, pressure fluctuations in the air along the propagation path of the laser beam [4].

Recent studies there are many ASER performance, average capacity performance for FSO systems is recently reported in [5]-[14]. In [5] Deng et al. presented analytic expressions and statistical models of the scintillation index and average capacity for multiple partial coherent beams propagating through strong turbulence MIMO FSO links. In [6] Sharma et al. evaluated the capacity of MIMO-OFDM/FSO with intersymbol interference (ISI) under strong atmospheric turbulence conditions. In [7] Kostas P. Peppas et al. capacity analysis of dual Amplify-and-Forward relayed FSO Systems over turbulence channels with pointing errors. In [8] Mona Aggarwal. Evaluated exact capacity of Amplify-and-Forward relayed optical wireless communication systems. In [14] H.D. Ai et al. Capacity analysis of amplify-and-forward SISI/FSO systems over atmospheric turbulence channels. However, to the best of our knowledge, the performance on average channel capacity of AF - MIMO/FSO systems for both weak and strong atmospheric turbulence channels has not been clarified. 
The remainder of the paper is organized as follows. In Section 2 the system descriptions are described in detail. In Section 3 atmospheric turbulence models are presented. In Section 4 channel capacity of AF-MIMO/FSO links is derived for weak and strong turbulence channels. In the Section 5 the numerical results are presented, analyzing the influence of turbulence strength, link distance, number relay stations and various MIMO configurations on the ASE of systems. The paper concludes with a summary given in the end.

\section{SYSTEM DESCRIPTIONS}

We consider a general AF-MIMO/FSO system using SC-QAM signals with $M$ transmitting lasers pointing toward an $N$ aperture receiver as depicted in Figure 1. Data transmission employing the same SC-QAM signal is transmitted with perfect synchronization by each of the $M$ transmit telescopes through an turbulence channel toward $N$ photo-detectors (PDs) with the light beam widths of each telescope assume to be wide enough to illuminate the entire receiver array. The transmitter's telescope array is assumed to produce the same total optical power irrespective of $M$ to enforce a fair comparison with the single transmitter case.

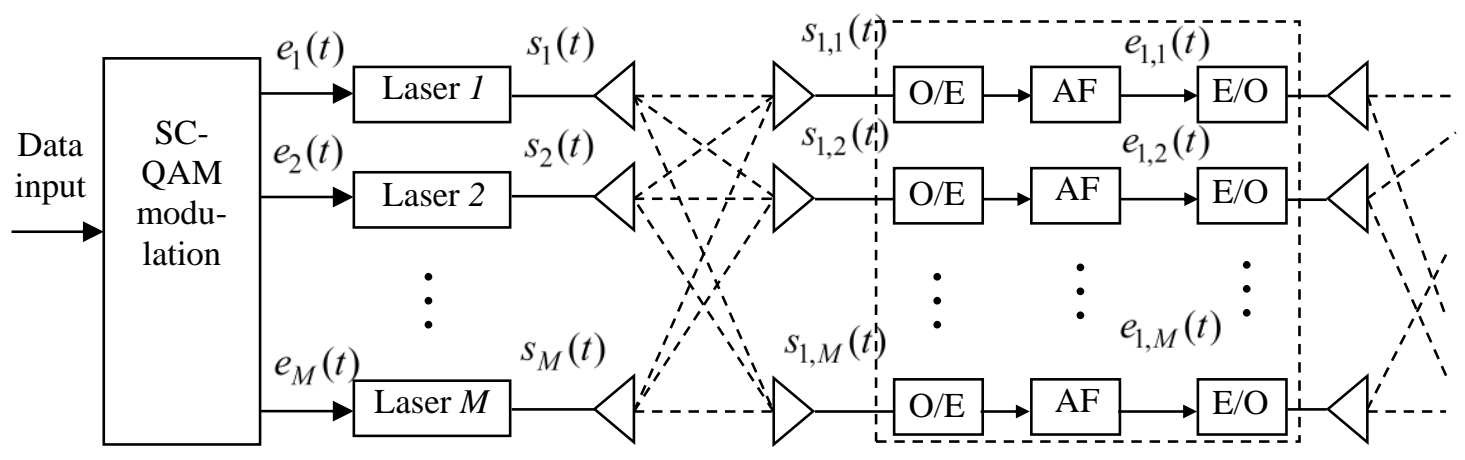

(a) Source node

(b) Relaying node $1^{\text {th }}$

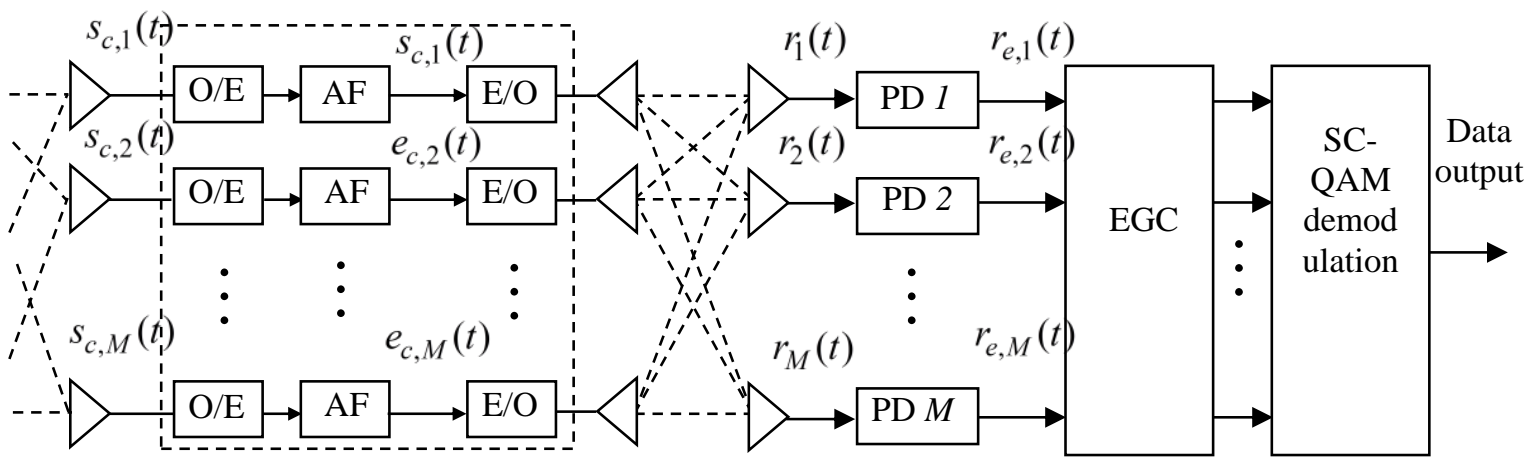

(c) relaying node $c^{\text {th }}$

(d) Destination node

Figure 1. The source node, relaying node, and destination node of AF - MIMO/FSO system using SC-QAM signals over atmospheric turbulence channel

The MIMO channel is modeled and can be denoted by an $M \times N$ matrix of the turbulence channel $\mathbf{X}=\left[X_{m n}(t)\right]_{m, n=1}^{M, N}$. The electrical signal at the output of the PD corresponding to the $n$th receive aperture output can be expressed as follows [9]

$$
r_{e}(t)=P_{s} e(t)\left[\sum_{m=1}^{M} \sum_{n=1}^{N} \prod_{i=0}^{C}\left(X_{i+1}\right)_{m n} \mathfrak{R}^{2 i+1} P_{i}\right]+\sum_{i=0}^{C}\left(\sum_{m=1}^{M} \sum_{n=1}^{N}\left(v_{m n}(t)\right)_{i}\right)
$$


where $e(t)$ represents the QAM signal, $X_{m n}(t)$ denotes the stationary random process for the turbulence channel between the $m$ th laser to the $n$th PD, $C$ is the number relay stations. $v_{m n}(t)$ is the AWGN with zero mean and variance $N_{0}^{(n)}$. Under the assumption of perfect channel estimation at the receiver side, the conditional input SNR at the PD can be written as a finite sum of sub-channel SNRs as

$$
\gamma=\left(\sum_{m=1}^{M} \sum_{n=1}^{N} \prod_{i=0}^{C}\left(\sqrt{\gamma_{i_{m n}}}\right)\right)^{2}
$$

where $\gamma_{m n}$ are RVs defined as the instantaneous electrical SNR at the output of the $n^{\text {th }}$ PD caused by signal from the $m^{\text {th }}$ laser. $\gamma_{m n}$ are given in the following

$$
\gamma_{m n}=\left(\frac{1}{M N} X_{m n} a \Re P_{s} \kappa\right)^{2} / N_{0}=\bar{\gamma}_{m n} X_{m n}^{2},
$$

in which, we denote $\gamma$ is the average SNR of systems.

$$
\gamma=\left(\frac{1}{M N} \kappa \Re^{2 i+1} P_{s} \sum_{m=1}^{M} \sum_{n=1}^{N} \prod_{i=1}^{C}\left(X_{i+1} P_{i}\right)\right)^{2} / N_{0}=\bar{\gamma}\left(\prod_{i=0}^{c} X_{i+1}\right)^{2} .
$$

\section{ATMOSPHERIC TURBULENCE MODELS}

There exist a few published papers concerning the probability density function (pdf) of multi-hop transmission over amplify-and-forward relay fading channels [9], [10]. With amplify-and-forward free-space optical systems using SC-QAM modulation, for weak atmospheric turbulence condition, the turbulence included fading is assumed to be a random process that follows that log-normal distribution [9], [10], whereas for strong turbulence conditions, a gamma-gamma distribution is used [11].

\subsection{The Log-Normal Turbulence Model of AF-MIMO/FSO Systems}

In log-normal fading channel, the probability density function (pdf) for an normalized irradiance with log-normal, $X_{m n} \geq 0$, is described as [9], [10]

$$
f_{X_{m n}}(X)=\frac{1}{(\mathrm{C}+1) X_{a}^{C+1} \sigma_{s} \sqrt{2 \pi}} \exp \left[-\frac{\left(\ln (X)+0.5 \sigma_{s}^{2}\right)^{2}}{2 \sigma_{s}^{2}}\right],
$$

where $\sigma_{s}^{2}=\exp \left(\psi_{1}+\psi_{2}\right)-1$ with $\psi_{1}$ and $\psi_{2}$ are respectively given by

$$
\psi_{1}=\frac{0.49 \sigma_{2}^{2}}{\left(1+0.18 d^{2}+0.56 \sigma_{2}^{12 / 5}\right)^{7 / 6}}, \psi_{2}=\frac{0.51 \sigma_{2}^{2}}{\left(1+0.9 d^{2}+0.62 \sigma_{2}^{12 / 5}\right)^{5 / 6}}
$$

In equation (6), $d=\sqrt{k D^{2} / 4 L}$, where $k=2 \pi / \lambda$ is the optical wave number, $L$ is the link distance in meters, $\lambda$ is the optical wavelength, and $D$ is the receiver aperture diameter of the PD. The parameter $\sigma_{2}^{2}$ is the Rytov variance and in this case, is expressed by [1]

$$
\sigma_{2}^{2}=1.23 C_{n}^{2} k^{7 / 6} L^{11 / 6}
$$

where $C_{n}^{2}$ stands for the strength of the atmospheric turbulence, which is the altitude dependent and it given by

$$
C_{n}^{2}(h)=0.00594\left(\frac{v}{27}\right)^{2}\left(10^{-5} h\right)^{10} \exp \left(\frac{h}{1000}\right)+C_{n}^{2}(0) \exp \left(-\frac{h}{1000}\right)+2.7 \times 10^{-6} \exp \left(-\frac{h}{1500}\right),
$$


where $h$ is the altitude in meters, $v$ is the wind speed in meters per second and $C_{n}^{2}(0)$ is the value of $C_{n}^{2}$ at the ground in $\mathrm{m}^{-3 / 2}$. $C_{n}^{2}$ varies from $10^{-17} \mathrm{~m}^{-3 / 2}$ to $10^{-13} \mathrm{~m}^{-2 / 3}$ for weak up to strong turbulence cases, respectively.

\subsection{The Gamma-gamma Turbulence Model of AF-MIMO/FSO Systems}

The pdf of gamma-gamma fading channel, $X_{m n} \geq 0$, the pdf for an normalized irradiance with gamma-gamma, $X_{m n} \geq 0$, is described as [11]

$$
f_{X_{m n}}(X)=\frac{2(\alpha \beta)^{\frac{\alpha+\beta}{2}}}{(C+1) \Gamma(\alpha) \Gamma(\beta)} X_{a}^{\frac{\alpha+\beta}{2}-C-1} \times K_{\alpha-\beta}\left(2 \sqrt{\alpha \beta X_{a}}\right),
$$

where $\Gamma(\alpha)$ is the Gamma function and $K_{\alpha-\beta}(\cdot)$ is the modified Bessel function of the second kind of order $(\alpha-\beta)$, while the parameters $\alpha$ and $\beta$ are directly related to atmospheric conditions through the following expressions:

$$
\alpha=\left[\exp \left(\psi_{1}\right)-1\right]^{-1}, \quad \beta=\left[\exp \left(\psi_{2}\right)-1\right]^{-1} .
$$

\section{AVERAGE CHANNEL CAPACITY}

An optical wireless channel is a randomly time-variant channel and the received instantaneous electrical signal-to-noise ratio (SNR) is a random variable. Thus, the channel capacity must be considered as a random variable, and its average value, known as average channel capacity, $\langle\bar{C}\rangle$. The ASE in bits/s/Hz if the frequency response of the channel is known. The average channel capacity is given by [12].

$$
\langle\bar{C}\rangle=\int_{\Gamma} \mathrm{B} \log _{2}(1+\gamma) \times f_{\Gamma}(\Gamma) d \Gamma,(\mathrm{bit} / \mathrm{s} / \mathrm{Hz}),
$$

where B is the channel's bandwidth and is the total channel SNR and $\Gamma=\left\{\gamma_{n m}, n=1, \ldots, N, m=1, \ldots, M\right\}$ is the matrix of the MIMO atmospheric turbulence channels. The joint pdf $f_{\Gamma}(\Gamma)$ can be reduced to a product of the first-order pdf of each element $\gamma_{n m}$. The pdfs of $\Gamma$ are respectively described in log-normal [9], [10] and gamma-gamma [11] distributions as follows

$$
\begin{aligned}
& f\left(\gamma_{m n}\right)=\frac{1}{2(\mathrm{C}+1) \gamma_{m n}^{C+1} \sigma_{s} \sqrt{2 \pi}} \exp \left(-\frac{\left(\ln \left(\frac{\gamma_{m n}}{\bar{\gamma}_{m n}}\right)+\sigma_{s}^{2}\right)^{2}}{8 \sigma_{s}^{2}}\right), \\
& f\left(\gamma_{m n}\right)=\frac{2(\alpha \beta)^{\frac{\alpha+\beta}{2}}}{(\mathrm{C}+1) \Gamma(\alpha) \Gamma(\beta)} \frac{\frac{\gamma}{m n}^{\frac{\alpha+\beta}{4}} \frac{C+1}{2}}{\bar{\gamma}} K_{\alpha-\beta}\left(2 \sqrt{\alpha \beta \sqrt{\frac{\gamma_{m n}}{\bar{\gamma}_{m n}}}}\right) .
\end{aligned}
$$

\subsection{Capacity of Log-Normal AF - MIMO/FSO Channels} expressed as

Using Equations 11 and 12, the ASE of a log-normal of AF-MIMO/FSO channel capacity can be

$$
\left\langle\frac{\bar{C}}{B}\right\rangle=\frac{1}{2(\mathrm{C}+1) \sigma_{s} \sqrt{2 \pi} \ln (2)} \int_{\Gamma} \frac{\ln \left(1+\gamma_{m n}\right)}{\gamma_{m n}^{c+1}} \times \exp \left(-\frac{\left(\ln \left(\frac{\gamma_{m n}}{\bar{\gamma}_{m n}}\right)+\sigma_{s}^{2}\right)^{2}}{8 \sigma_{s}^{2}}\right) d \gamma_{m n} .
$$


We can let the ASE of a log-normal of AF-MIMO/FSO channel without relay station $(C=0)$.

$$
\left\langle\frac{\bar{C}}{B}\right\rangle_{C=0}=\frac{1}{2 \sigma_{s} \sqrt{2 \pi} \ln (2)} \int_{\Gamma} \frac{\ln \left(1+\gamma_{m n}\right)}{\gamma_{m n}} \times \exp \left(-\frac{\left(\ln \left(\frac{\gamma_{m n}}{\bar{\gamma}_{m n}}\right)+\sigma_{s}^{2}\right)^{2}}{8 \sigma_{s}^{2}}\right) d \gamma_{m n}
$$

In Equation 15, using the equality $\ln (1+x)=\sum_{k=1}^{+\infty}(-1)^{k+1} x^{k} / k$ for $0 \leq x \leq 1$, and the scaled complementary error function $\operatorname{erfc}(x)=e^{x^{2}} \operatorname{erfc}(x)$, the integral of channel capacity without relay station $(C=0)$ can be transformed to the following summation [12] for the estimation of the average capacity, with has the following form

$$
\begin{aligned}
\left\langle\frac{\bar{C}}{B}\right\rangle_{C=0} & =C_{0}\left(\sum_{k=1}^{\infty} \frac{(-1)^{k+1}}{k}\left[\operatorname{erfc}\left(\sqrt{2} \sigma_{s} k+\frac{\Gamma_{m n}}{2 \sqrt{2} \sigma_{s}}\right)+\operatorname{erfc}\left(\sqrt{2} \sigma_{s} k-\frac{\Gamma_{m n}}{2 \sqrt{2} \sigma_{s}}\right)\right]+\frac{4 \sigma_{s}}{\sqrt{2 \pi}}\right. \\
& \left.+\Gamma_{m n} \times \exp \left(\frac{\Gamma_{m n}^{2}}{8 \sigma_{s}^{2}}\right) \operatorname{erfc}\left(-\frac{\Gamma_{m n}}{2 \sqrt{2} \sigma_{s}}\right)\right) .
\end{aligned}
$$

In Equation 17, $\Gamma_{m n}=\ln \left(\bar{\gamma}_{m n}\right)-\sigma_{s}^{2}$ with $\bar{\gamma}_{m n}$ is the instantaneous received SNR output of EGC detector, and $C_{0}=\exp \left(-\Gamma^{2} / 8 \sigma_{s}^{2}\right) / 2 \ln 2$. In order to evaluate the result of equation (16) we obtain a very accurate approximation for the evaluation of the average capacity [13], we should estimate the infinitely sum over $k$, from $k=1$ to $k=8$, with has the following form

$$
\begin{aligned}
\left\langle\frac{C}{B}\right\rangle_{C=0}= & C_{0}\left(\sum_{k=1}^{8} \frac{(-1)^{k+1}}{k}\left[\operatorname{erfc}\left(\sqrt{2} \sigma_{s} k+\frac{\Gamma_{m n}}{2 \sqrt{2} \sigma_{s}}\right)+\operatorname{erfc}\left(\sqrt{2} \sigma_{s} k-\frac{\Gamma_{m n}}{2 \sqrt{2} \sigma_{s}}\right)\right]+\frac{4 \sigma_{s}}{\sqrt{2 \pi}}+\right. \\
& \left.\Gamma_{m n} \times \exp \left(\frac{\Gamma_{m n}^{2}}{8 \sigma_{s}^{2}}\right) \operatorname{erfc}\left(-\frac{\Gamma_{m n}}{2 \sqrt{2} \sigma_{s}}\right)\right) .
\end{aligned}
$$

Substituting Equations 17 into 14 and integral calculate. The ASE of AF-MIMO/FSO channel with lognormal distribution model can be expressed as

$$
\left\langle\frac{\bar{C}}{B}\right\rangle=\frac{1}{(\mathrm{C}+1) \gamma_{m n}^{C}}\left\langle\frac{C}{B}\right\rangle_{C=0}+\frac{C}{C+1} \int_{\Gamma}\left\langle\frac{C}{B}\right\rangle_{C=0} \frac{1}{\gamma_{m n}^{C+1}} d \gamma_{m n}
$$

\subsection{Capacity of Gamma-gamma AF-MIMO/FSO Channels}

Substituting equation (13) into (11), the average capacity of a gamma-gamma of AF - MIMO/FSO channel can be given by

$$
\left\langle\frac{\bar{C}}{B}\right\rangle=\frac{2(\alpha \beta)^{\frac{\alpha+\beta}{2}}}{(\mathrm{C}+1) \Gamma(\alpha) \Gamma(\beta) \ln (2)} \frac{1}{\bar{\gamma}_{m n}} \int_{\Gamma} \ln \left(1+\gamma_{m n}\right) \times \gamma_{m n}^{\frac{\alpha+\beta}{4}-\frac{C+1}{2}} K_{\alpha-\beta}\left(2 \sqrt{\alpha \beta \sqrt{\frac{\gamma_{m n}}{\bar{\gamma}_{m n}}}}\right) d \gamma_{m n}
$$

Using the Meijer $G$-function, $G_{m n}^{p q}[\cdot]$, to express the logarithmic term of $\ln (1+x)=G_{2,2}^{1,2}\left[\left.x\right|_{1,0} ^{1,1}\right]$, and $K_{v}=\frac{1}{2} G_{0,2}^{2,0}\left[\left.\frac{x^{2}}{4}\right|_{\frac{v}{2},-\frac{v}{2}} ^{-,}--\right]$, the ASE of AF - MIMO/FSO channel with gamma-gamma distribution model can be given by 


$$
\left\langle\frac{\bar{C}}{B}\right\rangle=\frac{(\alpha \beta)^{\frac{\alpha+\beta}{2}}}{2 \pi(\mathrm{C}+1) \Gamma(\alpha) \Gamma(\beta) \ln (2)} \frac{1}{\bar{\gamma}_{m n}} \times G_{2,6}^{6,1}\left[\left.\frac{(\alpha \beta)^{2}}{16 \bar{\gamma}_{m n}}\right|_{\frac{\alpha-\beta}{4}, \frac{\alpha-\beta+2}{4},-\frac{\alpha-\beta}{4},-\frac{\alpha+\beta-2 C+2}{4},-\frac{\alpha+\beta-2 C+2}{4}} ^{-\frac{\alpha+\beta+2}{4},-\frac{\alpha+\beta-2 C+2}{4}+1}\right]
$$

\section{NUMERICAL RESULTS}

Using the above closed mathematical forms as shown in Equation 18 and 20, we can estimate the average capacity of AF - MIMO/FSO system over atmospheric turbulence channel. We use (18) for the case of weak turbulence with log-normal distribution model, while (20) is used for strong case with the gammagamma distribution. In the analysis below, we evaluate the average capacity for three different values of turbulence strength $C_{n}^{2}=10^{-15} \mathrm{~m}^{-2 / 3}, C_{n}^{2}=9 \times 10^{-15} \mathrm{~m}^{-2 / 3}$, and $C_{n}^{2}=3 \times 10^{-14} \mathrm{~m}^{-2 / 3}$, link distance $L$ has been chosen to be $L=2000 \mathrm{~m}, \mathrm{~L}=4000 \mathrm{~m}$, different values of number relay stations $C=0, C=1$, and $C=2$ and amplification gain $P_{A F}=2 \mathrm{~dB}$. Assumes that, the relay stations are placed equidistant, turbulence conditions between relay of stations is the same. Relevant parameters considered in our analysis are provided in Table 1.

Table 1. Relevant Parameters Considered in Our Analysis

\begin{tabular}{lcc}
\hline \multicolumn{1}{c}{ Parameter } & Symbol & Value \\
\hline Laser Wavelength & $\lambda$ & $1550 \mathrm{~nm}$ \\
Photodetector responsivity & $\Re$ & $1 \mathrm{~A} / \mathrm{W}$ \\
Modulation Index & $\kappa$ & 1 \\
Total noise variance & $N_{O}$ & $10^{-7} \mathrm{~W} / \mathrm{Hz}$ \\
Receiver aperture diameter of the PD & $\mathrm{D}$ & $0.08 \mathrm{~m}$ \\
In-phase/quadrature signal amplitudes & $M_{I} \times M_{Q}$ & $8 \times 4$ \\
Amplification gain & $P_{A F}$ & $2 \mathrm{~dB}$ \\
\hline
\end{tabular}

In Figures 2-3, illustrate the ASE of different AF - MIMO/FSO channels (i.e., SISO, $2 \times 2$ and $4 \times 4$ MIMO/ FSO channels) with respect to $\bar{\gamma}$, for three values of the relay stations $C,(C=0, C=1$, and $C=2)$ with two different link distances $\mathrm{L}=2000 \mathrm{~m}$ and $\mathrm{L}=4000 \mathrm{~m}$, amplification gain $P_{A F}=2 \mathrm{~dB}$, for strong atmospheric turbulence, $C_{n}^{2}=3 \times 10^{-14} \mathrm{~m}^{-2 / 3}$. It can be also seen that the ASE strongly depends on MIMO configurations, at achieve the $\mathrm{SNR}=10 \mathrm{~dB}$ for MIMO configurations $\mathrm{M}=\mathrm{N}=4, \mathrm{C}=0, \mathrm{ASE}=7 \mathrm{~b} / \mathrm{s} / \mathrm{Hz}$ with $\mathrm{L}=2000 \mathrm{~m}$ but $\mathrm{L}=4000 \mathrm{~m} \mathrm{ASE}=6 \mathrm{~b} / \mathrm{s} / \mathrm{Hz}$. Similar, for $\mathrm{M}=\mathrm{N}=2$ the corresponding result is $\mathrm{ASE}=5 \mathrm{~dB}$ with and $\mathrm{ASE}=4 \mathrm{~dB}$ with $\mathrm{L}=4000 \mathrm{~m}$. For the case $\mathrm{C}=1$ and $\mathrm{C}=2$ the results are similar, $\mathrm{ASE}$ decreases $1 \mathrm{~dB}$. The ASE strongly depends on the number relay stations, with $\mathrm{L}=2000 \mathrm{~m}, \mathrm{M}=\mathrm{N}=4$ when number of relay stations increases from $\mathrm{C}=0$ to $\mathrm{C}=1$ and from $\mathrm{C}=1$ to $\mathrm{C}=2 \mathrm{ASE}$ of systems decreases $3 \mathrm{~dB}$ and $1.5 \mathrm{~dB}$, respectively to achieve the $\mathrm{SNR}=10 \mathrm{~dB}$. The ASE strongly depends on the link distance gets longer, with $\mathrm{C}=0, \mathrm{M}=\mathrm{N}=4$, when increasing distance from $\mathrm{L}=2000 \mathrm{~m}$ to $\mathrm{L}=4000 \mathrm{~m}$ ASE of systems decreases $1 \mathrm{~dB}$. The impact of SNR on the ASE of systems is more significant in low regions than in high regions. The reason of this is that as optical link distance gets longer, the signal propagates in the atmospheric with longer distances. It has been that observed that with increase in the value of $\mathrm{C}$, capacity performance of the system deteriorates.

Figures 4-5, illustrate the ASE of different AF-MIMO/FSO channels (i.e. SISO, $2 \times 2$ and $4 \times 4$ MIMO/ FSO channels) with respect to $\bar{\gamma}$, for three values of the turbulence strength $C_{n}^{2}$, number of relay stations $C=1$ with two different link distances $\mathrm{L}=2000 \mathrm{~m}$ and $\mathrm{L}=4000 \mathrm{~m}$, amplification gain $P_{A F}=2 \mathrm{~dB}$. It can be also seen that the ASE strongly depends on the atmospheric turbulence strength, at link distance $\mathrm{L}=4000 \mathrm{~m}, \mathrm{M}=\mathrm{N}=4$, values of turbulence strength increases from $C_{n}^{2}=10^{-15} \mathrm{~m}^{-2 / 3}$ to $C_{n}^{2}=9 \times 10^{-15} \mathrm{~m}^{-2 / 3}$ and from $C_{n}^{2}=9 \times 10^{-15} \mathrm{~m}^{-2 / 3}$ to $C_{n}^{2}=3 \times 10^{-14} \mathrm{~m}^{-2 / 3} \mathrm{ASE}$ of systems decreases $0.3 \mathrm{~dB}$ and $0.4 \mathrm{~dB}$, respectively to achieve the $\mathrm{SNR}=20 \mathrm{~dB}$. The ASE strongly depends on number relay stations, and as the link distance gets longer, the ASE under the weak turbulence conditions is higher than in the cases of moderate and strong turbulence, especially with longer link distance $L$. The reason of this is that as optical link distance gets longer, the signal propagates in the atmospheric with longer distances; the influence of the turbulence therefore becomes stronger. On the other hand, as expected, the ASE could be improved by approximately 2 $(\mathrm{b} / \mathrm{s} / \mathrm{Hz})$ when the system is upgraded from SISO/FSO to $2 \times 2 \mathrm{MIMO} / \mathrm{FSO}$ or from $2 \times 2 \mathrm{MIMO} / \mathrm{FSO}$ to $4 \times 4$ MIMO/FSO for number of relay stations $C=0$, approximately $1(\mathrm{~b} / \mathrm{s} / \mathrm{Hz})$ for $C=1$, and approximately 0.7 (b/s/Hz) for $C=2$. 


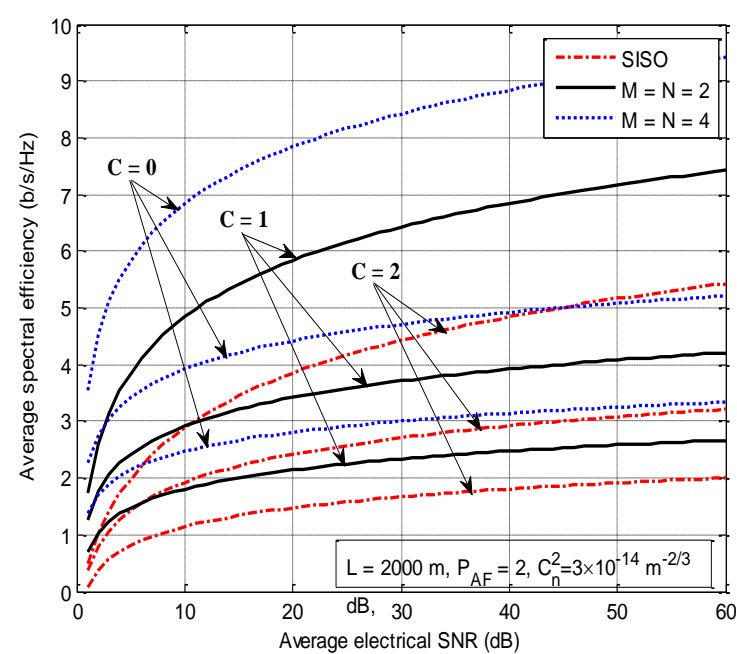

Figure 2. ASE versus the average electrical SNR of various AF-MIMO/FSO channels and different number of relay station for strong atmospheric turbulence strengths under link distance $\mathrm{L}=2000 \mathrm{~m}$

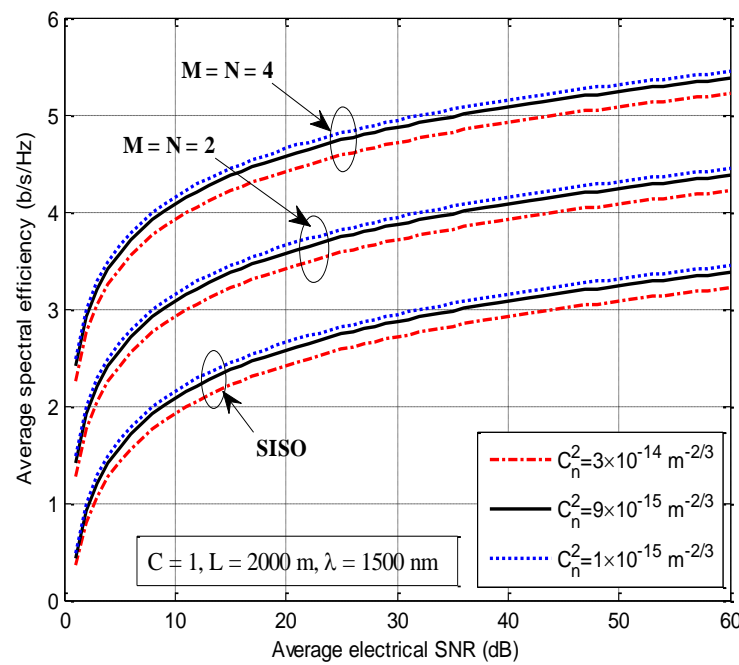

Figure 4. ASE versus the average electrical SNR of different AF-MIMO/FSO channels for weak to strong atmospheric turbulence strengths under link distance $\mathrm{L}=2000 \mathrm{~m}$, number of relay stations $\mathrm{C}=1$

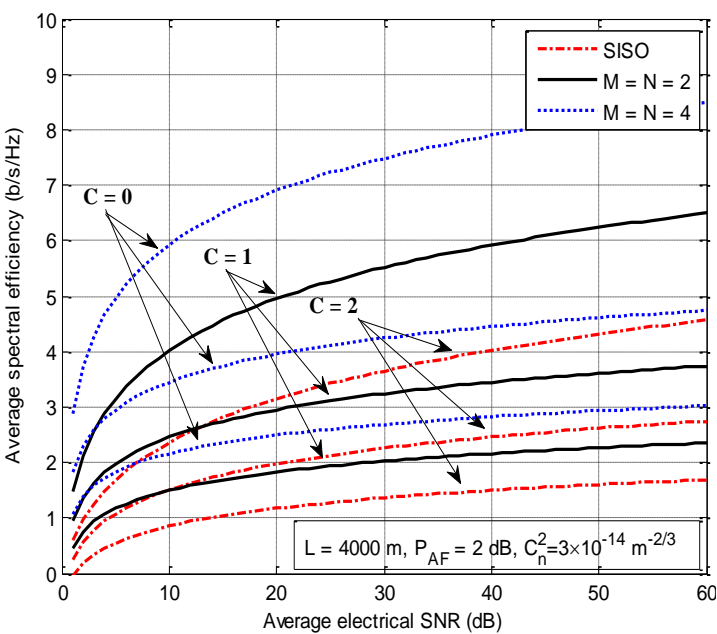

Figure 3. ASE versus the average electrical SNR of various AF-MIMO/FSO channels and number relay station for strong atmospheric turbulence under link distance $\mathrm{L}=4000 \mathrm{~m}$

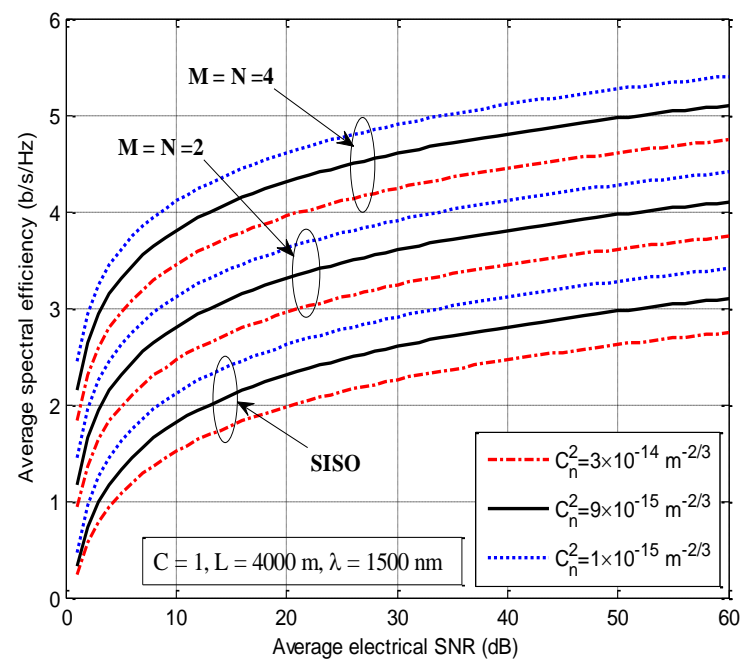

Figure 5. ASE versus the average electrical SNR of different AF - MIMO/FSO channels for weak to strong atmospheric turbulence strengths under link distance $\mathrm{L}=4000 \mathrm{~m}$, number of relay stations $\mathrm{C}=1$

Figures 6-7, illustrate the ASE of different AF-MIMO/FSO channels versus number of relay stations for three values of the turbulence strength under free-space two different link distances $\mathrm{L}=2000 \mathrm{~m}$ and $\mathrm{L}=4000 \mathrm{~m}$, amplification gain $P_{A F}=2 \mathrm{~dB}$. It is clearly shown that the ASE performance is improved significantly with the increase of number of lasers and receivers and the ASE performance is decreases significantly with the increase of number of relay stations. In addition, as expected, ASE increases as number of lasers $M$ and receiver $N$ increase from SISO to $2 \times 2$ MIMO and $4 \times 4$ MIMO. It can be also seen that the ASE strongly depends on the atmospheric turbulence strength, MIMO configurations, and as the link distance gets longer. Increasing the number of relay stations, capacity performance of the system deteriorates but increases the gain of power, this problem is very important in the FSO system. 


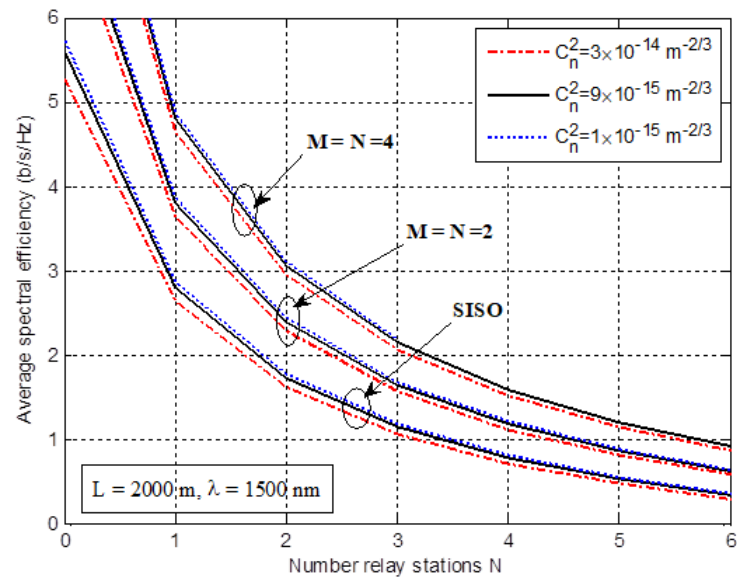

Figure 6. ASE versus the number of relay stations of FSO channels for weak to strong atmospheric turbulence strengths, amplification gain under link distance $\mathrm{L}=2000 \mathrm{~m}$

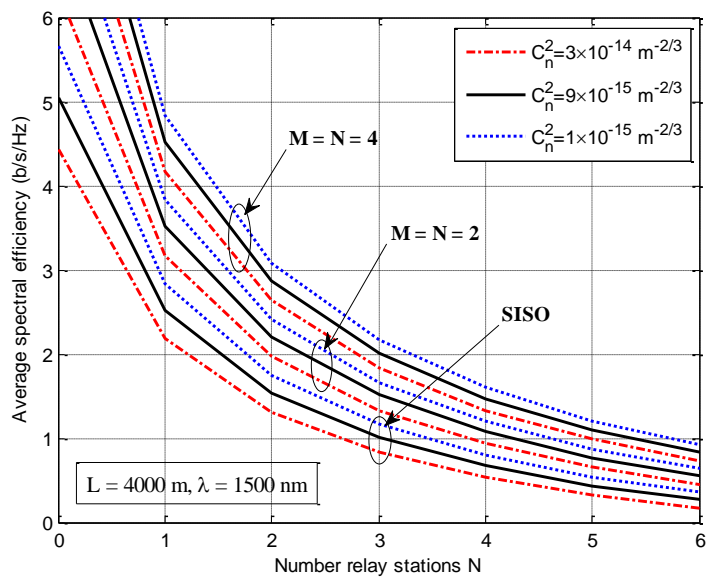

Figure 7. ASE versus the number relay stations of FSO channels for weak to strong atmospheric turbulence strengths, amplification gain $P_{A F}=2 \mathrm{~dB}$ under link distance $\mathrm{L}=4000 \mathrm{~m}$

\section{CONCLUSION}

The purpose of this paper is to present the performance analysis on the average channel capacity of $\mathrm{AF}$ - MIMO/FSO systems using SC-QAM signals over atmospheric turbulence channels. The log-normal and gamma-gamma distribution models were used to describe the fluctuation of the optical propagating over atmospheric turbulence channels. The ASE is calculated based on the probability density function of the FSO system combining the relay stations, ASE considering different link conditions, number of relay stations and MIMO configurations. The numerical results showed that, with the similar link distance and turbulence strength, regardless the link distance and turbulence condition, the ASE of the FSO link could be improved by approximately $2(\mathrm{~b} / \mathrm{s} / \mathrm{Hz})$ when the system is upgraded from from SISO/FSO to $2 \times 2 \mathrm{MIMO} / \mathrm{FSO}$ or from $2 \times 2 \mathrm{MIMO} / \mathrm{FSO}$ to $4 \times 4 \mathrm{MIMO} / \mathrm{FSO}$ for number of relay stations $C=0$, approximately $1(\mathrm{~b} / \mathrm{s} / \mathrm{Hz})$ for $C=1$, and approximately $0.7(\mathrm{~b} / \mathrm{s} / \mathrm{Hz})$ for $C=2$. The ASE strongly depends on the atmospheric turbulence strength, MIMO configurations, number of relay stations and as the link distance. Increasing the number of relay stations, capacity performance of the system deteriorates but increases the gain of power, this problem is very important in the FSO system.

\section{REFERENCES}

[1] A.K. Majumdar. Free-space laser communication performance in the atmospheric channel. L. Opt. Fiber Commun. Rep. 2005; 2; 345-396.

[2] S. Arnon. Optical wireless communications. in Encyclopedia of Optical Engineering. New York: Marcel Dekker, 2003; 1866-1886.

[3] D. Kedar and S. Arnon. Urban optical wireless communication networks: The main challenges and possible solutions. IEEE Commun. Mag. 42(5); 2-7.

[4] X. Zhu and J. M. Kahn. Free-space optical communication through atmospheric turbulence channels. IEEE Trans. Commun. 2002; 50(8); 1293-1300.

[5] Huiying Zhang, Hongzuo Li, Xiao Dongya, Cai Chao. Performance Analysis of Different Modulation Techniques for Free-Space Optical Communication System. TELKOMNIKA JOURNAL. 2015; 13(3); 880-888.

[6] Rekawt Sabir Hassan, T. A. Rahman, A. Y. Abdulrahman. LTE Coverage Network Planning and Comparison with Different Propagation Models. TELKOMNIKA JOURNAL. 2015; 12(1); 153-162

[7] Kostas P. Peppas, Argyris N. Stassinakis, Hector E. Nistazakis, and George S. Tombras. Capacity Analysis of Dual Amplify-and-Forward Relayed Free-Space Optical Communication Systems over Turbulence Channels with Pointing Errors. J. OPT. COMMUN. NETW. 2013; 5(9).

[8] Mona Aggarwal. Exact Capacity of Amplify-and-Forward Relayed Optical Wireless Communication Systems. IEEE Photonics Technology Letters. 2015; 27(8).

[9] Duong Huu Ai, Do Trong Tuan, and Ha Duyen Trung. Pointing Error Effects on Performance of Amplify-andForward Relaying MIMO/FSO Systems Using SC-QAM Signals over Log-Normal Atmospheric Turbulence Channels. The 8th Asian Conference on Intelligent Information and Database Systems. 2016; 607-619.

[10] Ha Duyen Trung and Do Trong Tuan. Performance of Amplify-and Forward Relaying MIMO Free-Space Optical Systems over Weak Atmospheric Turbulence Channels. The Second NAFOSTED Conference on Information and Computer Science. 2015; 223-228. 
[11] Duong Huu Ai, Ha Duyen Trung, and Do Trong Tuan. AF Relay-Assisted MIMO/FSO/QAM Systems in GammaGamma Fading Channels. The Third NAFOSTED Conference on Information and Computer Science. 2016; 147152.

[12] Ha Duyen Trung, Duong Huu Ai, and Anh T. Pham. Average Channel Capacity of Free-Space Optical MIMO Systems over Atmospheric Turbulence Channels. ASEAN Engineering Journal. 2015; 5(2); 57-66.

[13] Amine Laourine. Estimating the Ergodic Capacity of Log-Normal Channels. IEEE Communications Letters. 2007; 11(7).

[14] Duong Huu Ai et al. Capacity analysis of amplify-and-forward free-space optical communication systems over atmospheric turbulence channels. The Seventh International Conference on Information Science and Technology (ICIST). 2017; 103-108.

\section{BIOGRAPHY OF AUTHOR}

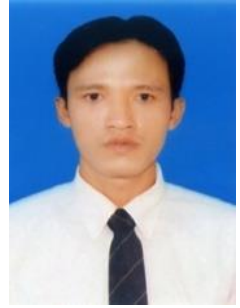

Duong Huu Ai, received M.E degree of Electronic engineering from Danang University of Technology, Vietnam, in 2011. Currently, he is a lecturer at the Vietnam Korea friendship Information Technology College and $\mathrm{PhD}$ student at Hanoi University of Science and Technology. His research interests include optical wireless communications, radio over fiber, and broadband networks. 